\title{
Control of Long Pulse Pumped Supercontinuum Generation Using Weak Trigger Signal
}

\author{
Chao Huang, Student Member, OSA, K. Nakkeeran, Senior Member, IEEE and Senior Member, OSA and Qian \\ $\mathrm{Li}^{*}$, Senior Member, OSA and Member, IEEE
}

\begin{abstract}
Through numerical simulations, we study the effects of weak continuous wave $(\mathrm{CW})$ or weak pulse triggering on the picosecond (ps) pulse pumped supercontinuum (SC) generation. To actively improve the spectral broadening and control both phase and intensity stability of the SC generation, we fix the frequency shift of the trigger pulse (with respect to the pump signal) with different durations and then tune the peak power. Detailed simulation results show that either a weak $\mathrm{CW}$ or weak pulse trigger can greatly improve the spectral broadening of $\mathrm{SC}$ spectrum generated by a ps pulse pump. Compared to weak CW trigger, a weak femtosecond (fs) pulse or ps pulse trigger can assist the generation of SC with much better coherence and SNR, but precise control of the time delay between the pump and trigger is required.
\end{abstract}

Index Terms-supercontinuum generation, nonlinear optics, optical fibers, modulation instability

\section{INTRODUCTION}

$\mathbf{S}$ UPERCONTINUUM (SC) generation based on optical fibers has attracted great interests in both research studies and various real life applications in the past decades. With a remarkable feature of ultrawideband optical spectrum, SC generation in optical fibers has been implemented in a wide range of applications, such as flattened Raman amplifiers, generation of ultrashort optical pulses, optical frequency metrology, and optical coherence tomography (OCT) [1]-[4]. SC generation can be obtained through bulk media, optical fibers as well as photonic crystal fibers (PCFs). The starting point pump source for SC generation can be a femtosecond (fs), picosecond (ps), or nanosecond (ns) pulse to even continuous wave (CW). The stability of the light sources is a vital requirement for the generation of good quality SC signal. The SC signal noise characteristics remain as a main problem and attracted more research studies for improvement. Prior studies have revealed that fs pulse pump can enhance the stability of SC generation. But SC generation through ultrashort pulse needs exceptional dispersion control and is continually sensitive to perturbations. Many research studies continued to focus on the long pulse (ps, ns or $\mathrm{CW}$ ) pumped SC generation where modulation instability (MI) plays an important role in the spectral broadening [5]-[8]. SC signal generated using long pulse pump finds application

This work was supported by National Natural Science Foundation of China under Grants 61675008, and Shenzhen Science and Technology Innovation Commission under Grant GJHZ20180411185015272. (Corresponding authors: Qian Li.) C. Huang and Q. $\mathrm{Li}$ are with the School of Electronic and Computer Engineering, Peking University, Shenzhen 518055, China (e-mail: liqian@ece.pku.edu.cn). K Nakkeeran is with the School of Engineering, Fraser Noble Building, University of Aberdeen, Aberdeen AB24 3UE, UK (e-mail: k.nakkeeran@abdn.ac.uk). in various fields, such as photon counting detection system [9], excited state spectroscopy [10], fluorescence life-time and multi-photon imaging [11] and coherent anti-Stokes Raman scattering microscopy [12].

The dominating effect in the process of SC generation via long pulse pump is the MI, which is originated from random noise. This noise induced MI signal forms the starting point of the SC signal and hence the subsequent evolution that forms further spectral broadening of the SC signal is unstable [13]. The instability speeds up the growth of sidebands in the spectral evolution that leads the SC signal to be poor in both phase correlation and intensity stability [14], [15]. Also extreme form of amplified noise can induce optical rogue waves in the generation of SC signal [16]. Furthermore, a series of studies on SC generation reported that the optical rogue waves are formed by a noise-sensitive nonlinear process and the statistics shows that its characteristics is an L shaped distribution [16]-[20]. Several effective control methods were reported to manipulate the generation of SC using the long pulse pump source, such as, CW triggering mechanism, a coherent pulse seed, a solid-core photonic bandgap fiber or a sliding frequency filter [7], [21]-[24]. Numerical simulation results showed that a weak $\mathrm{CW}$ trigger could control the process of ps pulse pumped SC generation [22].

To the best of our knowledge, previous studies that are concentrating on the control of optical rogue waves for ps pulse pumped SC generation in optical fiber [25]-[27] did not systematically discuss the effects of different duration triggers for optical fiber SC generation. In this work, we investigate how to improve the spectral broadening and stability of the ps pulse pumped SC generation. By adding a weak trigger signal such as fs pulse, ps pulse or $\mathrm{CW}$ to the ps pump pulse, we can control the formation of optical rogue waves in the $\mathrm{SC}$ generation. The frequency shift (with respect to the pump signal) and peak power of the weak trigger signal are two major influence factors of the ps pulse pumped SC generation. So we first fix the frequency shift that can lead to the best performance for the spectral broadening and coherence of the generated SC signal. Then by adjusting the peak power of different duration of trigger signal, we compare the spectral broadening and coherence of the resulting SC to figure out which kind of trigger signal can achieve the best control of the optical rogue waves in the ps pulse pumped SC generation.

\section{Theoretical Model}

SC generation in optical fibers can be modelled by the generalized nonlinear Schrodinger equation (GNLSE) [2]. It 
has been reported that the simulation results obtained from GNLSE and experimental results are in very good agreement [2], [5], [13]. Recently, our experimental results on the CWtriggered SC generation in highly nonlinear fibers agree well with our simulation results obtained from the GNLSE model [28].

$$
\begin{array}{r}
\frac{\partial A}{\partial z}+\frac{\alpha}{2} A+i \frac{\beta_{2}}{2} \frac{\partial^{2} A}{\partial t^{2}}-\frac{\beta_{3}}{6} \frac{\partial^{3} A}{\partial t^{3}}=i \gamma \times\left(1+i \tau_{\text {shock }} \frac{\partial}{\partial t}\right) \\
\times\left[A(z, t) \int_{-\infty}^{+\infty} R\left(t^{\prime}\right)\left|A\left(z, t-t^{\prime}\right)\right|^{2} d t^{\prime}\right]
\end{array}
$$

where $A(z, t)$ represents the slowly varying amplitude of the pulse envelope, $z$ and $t$ are the propagation distance and the propagation time respectively. Here, the loss, second-order dispersion, third-order dispersion and nonlinear coefficients of the fiber are $\alpha, \beta_{2}, \beta_{3}$ and $\gamma$. The dispersion of the nonlinearity is related with the optical shock formation, which depicted by $\tau_{\text {shock }}=1 / \omega_{0}$, where $\omega_{0}$ is the center frequency. Raman response function $R(t)=\left(1-f_{\mathrm{R}}\right) \delta(t)+f_{\mathrm{R}} h_{\mathrm{R}}(t)$, where $h_{\mathrm{R}}$ can be determined by the fused silica experimentally. In addition, $f_{\mathrm{R}}=0.18$ is the fractional contribution of the delayed Raman response and $\delta(t)$ is the Dirac delta function. The GNLSE may be extended to include birefringence effects through coupled mode equations. Mostly in experiments [28], optical polarizers are often utilized to ensure that the polarization of both the pump and trigger signals are at the same state, in the theoretical model as well GNLSE with single state of polarization assumption is considered.

A chirp-free Gaussian shape pulse $\sqrt{P_{0}} \exp \left(-t^{2} / 2 / T_{0}^{2}\right)$ at a center wavelength of $\lambda_{0}=1554.5 \mathrm{~nm}$ is launched into a 50 meters long highly nonlinear dispersion shifted fiber (HNL-DSF) with zero dispersion wavelength of $1554 \mathrm{~nm}$. This pump pulse duration is set to be $T_{0}=4 \mathrm{ps}$ (FWHM $=6.66 \mathrm{ps}$ ). The peak power of the input pulse is set to be $P_{0}=60 \mathrm{~W}$. Input pulse shot noise is modeled semiclassically through the addition of noise seed of one-photonper-mode with random phase on each spectral discretization bin [2]. And the scale of the noise amplitude is assumed to be $10^{-6}$ of the pump amplitude. The second order and third order dispersion coefficients at the pump wavelength are $\beta_{2}=-0.0224 \mathrm{ps}^{2} / \mathrm{km}, \beta_{3}=0.0576 \mathrm{ps}^{3} / \mathrm{km}$ and the nonlinear coefficient $\gamma=14 / \mathrm{W} / \mathrm{km}$. For the pulse duration of weak fs pulse trigger or ps pulse trigger, we have investigated the performance of the SC generation for two different ranges of the trigger pulse duration range (i) $10 \mathrm{fs}$ to $100 \mathrm{fs}$ at an interval of $10 \mathrm{fs}$ for femtosecond order trigger pulse duration and (ii) 1 ps to 10 ps at an interval of 1 ps for picosecond order trigger pulse duration. The simulation results showed that both the fs pulse trigger and ps pulse trigger can greatly improve the bandwidth and coherence of the output SC if the trigger pulse frequency position is chosen at an appropriate position relative to that of the pump. In this paper we assumed the duration of fs pulse trigger and ps pulse trigger to be $T_{s}=100 \mathrm{fs}$ $(\mathrm{FWHM}=166.5 \mathrm{fs})$ and $T_{s}=4 \mathrm{ps}(\mathrm{FWHM}=6.66 \mathrm{ps})$ respectively. These trigger pulse durations are similar to those reported in the recent experimental research work [5]. The instantaneous amplitude pulse trigger is considered in the form of $\sqrt{\varepsilon P_{0}} \exp \left[i \Omega\left(t+t_{\mathrm{d}}\right)\right] \exp \left[-\left(t+t_{\mathrm{d}}\right)^{2} / 2 / T_{s}^{2}\right]$, where $\varepsilon$ is the fractional amount of the trigger intensity compared with the pump power $\left(P_{0}\right), \Omega$ is the frequency of the trigger signal, the weak trigger pulse is delayed in time with respect to the pump pulse for a value of $t_{\mathrm{d}}, T_{s}$ is the duration of the pulse trigger. $\varepsilon$ and $\Omega$ are varied for the investigation of the best spectral broadening, SNR and coherence of the SC generation. For better spectral broadening, previous studies have demonstrated both experimentally and theoretically that the SC bandwidth reaches its maximum when the trigger pulse wavelength is near to the MI gain peak [21], [22]. In our example, as shown in Fig. 1(b), the MI gain peaks are located at $1475.4 \mathrm{~nm}$ and $1645.4 \mathrm{~nm}$, that corresponds to frequency shifts of -65 $\mathrm{THz}$ and $67 \mathrm{THz}$, from the pump signal. We also carried out numerical simulations by varying the frequency shift from $150 \mathrm{THz}$ to $150 \mathrm{THz}$ at an interval of $10 \mathrm{THz}$. One hundred simulations were performed with different initial random noise for each frequency shift, and the resulted SC signal indicated that the frequency shift range from $60 \mathrm{THz}$ to $80 \mathrm{THz}$ could achieve relatively broad spectra. Both positive and negative frequency shifts resulted in almost same value for the SC signal bandwidth. We also carried out numerical simulations with a finer interval of $1 \mathrm{THz}$ within the frequency shift range from $60 \mathrm{THz}$ to $80 \mathrm{THz}$ and from the resulted SC signal, here we reported the results for a frequency shift of $67 \mathrm{THz}$ as maximum bandwidth was obtained for this value. In order to improve the intensity stability of the output SC, we varied the frequency shift from $-150 \mathrm{THz}$ to $150 \mathrm{THz}$ at an interval of $10 \mathrm{THz}$ and performed 100 simulations with different initial random noise for each frequency shift. The simulation results showed that the range of frequency shift from $50 \mathrm{THz}$ to 70 $\mathrm{THz}$ could improve the intensity stability of the SC. We also performed numerical simulations with a finer interval of $1 \mathrm{THz}$ within the frequency shift range from $50 \mathrm{THz}$ to $70 \mathrm{THz}$ and found that the best frequency shift for best intensity stability occurs at $\Omega=60 \mathrm{THz}$. Therefore, the frequency shift of the fs pulse trigger, ps pulse trigger or $\mathrm{CW}$ trigger with respect to the center wavelength of the pump signal is selected to be $\Omega=67 \mathrm{THz}$ (better bandwidth) or $\Omega=60 \mathrm{THz}$ (better coherence and SNR). For the better performance (to enhance the stoke side of the simulated Raman scattering) we add the weak trigger signal to the red side of center wavelength of pump. For the fs or ps pulse trigger, a time delay between trigger and pump pulses is provided to enhance the spectral broadening mechanisms that are due to the delayed processes. The weak pulse trigger is delayed in time with respect to the pump pulse for a value of $t_{\mathrm{d}}$. We have investigated the range of time delay from -10 ps to 10 ps at an interval of 1 ps and performed 100 simulations for each time delay to figure out the appropriate time delay that can achieve SC with best possible bandwidth and high intensity stability (high coherence). The simulation results indicated that SC generated with 3 ps time delay for the trigger pulse has the maximum bandwidth and highest coherence, and hence $3 \mathrm{ps}$ is considered as the value of $t_{\mathrm{d}}$ for all simulations performed for both fs or ps pulse trigger. Considering the $\mathrm{CW}$ trigger in the form of $\sqrt{\varepsilon P_{0}} \exp (i \Omega t)$, it is not necessary to consider the time delay between the pump 

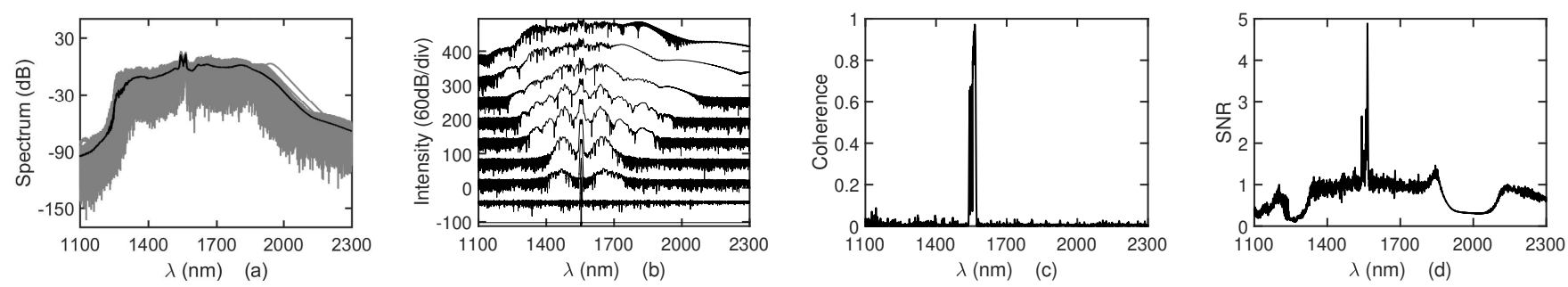

Fig. 1. (a) Spectra, (b) spectral evolution, (c) coherence and (d) SNR of ps pulse pumped SC generation without any trigger pulse. To achieve best SC signal, 100 different simulations (separately for spectra and coherence) with different noise seeds are utilized. Each curve in Fig. 1(b) (from the bottom to the top) represents the spectrum at $0 \mathrm{~m}, 5 \mathrm{~m}, 15 \mathrm{~m}, 23 \mathrm{~m}, 30 \mathrm{~m}, 32 \mathrm{~m}, 35 \mathrm{~m}, 40 \mathrm{~m}, 50 \mathrm{~m}$.
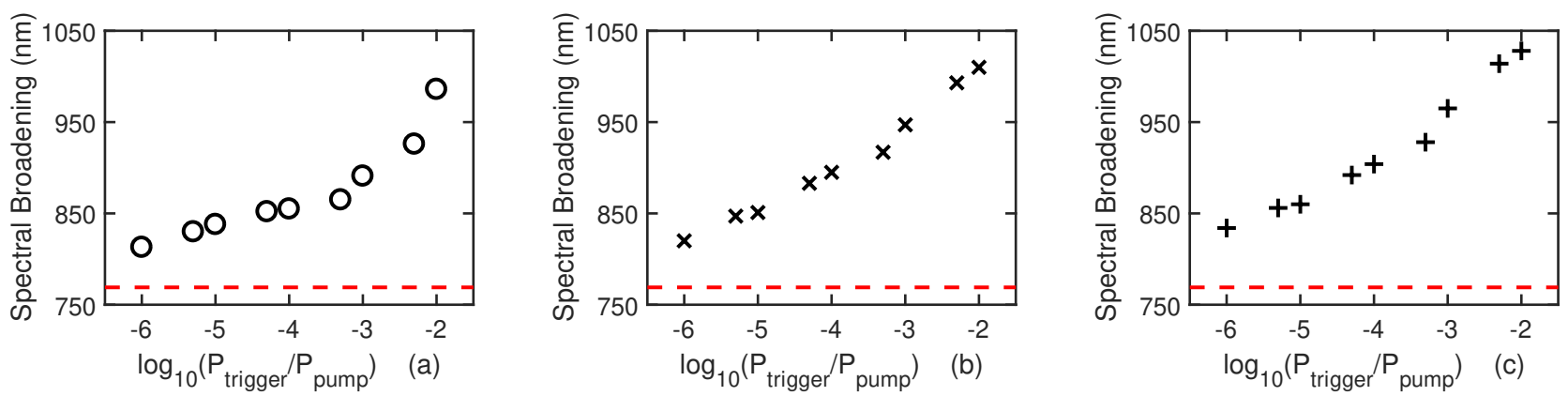

Fig. 2. Comparison of spectral broadening between (a) 100 fs-triggered (circle marks), (b) 4 ps-triggered (cross marks), (c) CW-triggered (plus marks) and untriggered (red dashed line) SC generation. The peak power of triggers ranges from $0.0001 \%$ to $1 \%$ of the peak power of pump pulse.

and CW-trigger.

\section{Simulation Results}

We systematically present the detailed results of the numerical simulations of ps pulse pumped SC generation with weak different duration trigger signals in terms of bandwidth, coherence and SNR. We first simulate the situation with only the ps pump pulse without any addition of any weak trigger signal. Then, we perform the simulation with trigger signal and tune the frequency shift to investigate the best frequency for the trigger in terms of best spectral broadening and coherence. Finally we vary the peak power and the duration trigger signal to investigate which kind of trigger can achieve the best control of the optical rogue waves in the ps pulse pumped $\mathrm{SC}$ generation.

\section{A. Evolution and characteristics of untriggered ps pulse pumped SC generation}

To illustrate how different duration trigger signals can affect the evolution and characteristics of ps pulse pumped SC generation, we first report the evolution and characteristics of ps pulse pumped SC without any trigger signal. Figure 1(a) shows the spectra of the untriggered ps pulse pumped SC generation. One hundred simulations are carried out with different random noise seeds while keeping other parameters same. The gray lines stand for the spectral output of one hundred simulations and the black line stands for the calculated mean in figure 1(a). Here, we consider $-50 \mathrm{~dB}$ as a standard line for spectral output. We can figure out that the spectrum of the untriggered ps pulse pumped SC signal spans from $1276 \mathrm{~nm}$ to 2045 $\mathrm{nm}$ with a bandwidth equals to $769 \mathrm{~nm}$. Figure 1(b) shows the generation of untriggered ps pulse pumped SC where MI induced by random noise seeds acts as the initial source of the spectral broadening. We reported the spectral evolution of the ps pulse pumped SC generation without any trigger pulse in a fiber span of $50 \mathrm{~m}$ in Fig. 1(b) (curves from the bottom to the top represents the spectrum of the SC signal at fiber lengths of $0 \mathrm{~m}, 5 \mathrm{~m}, 15 \mathrm{~m}, 23 \mathrm{~m}, 30 \mathrm{~m}, 32 \mathrm{~m}, 35 \mathrm{~m}$, $40 \mathrm{~m}, 50 \mathrm{~m}$ respectively). As shown in figure 1(b), we can find the MI peak is located at $-65 \mathrm{THz}$ and $67 \mathrm{THz}$ while the corresponding wavelengths are $1475.4 \mathrm{~nm}$ and $1645.4 \mathrm{~nm}$, respectively. Previous work has shown that if the weak trigger is added at the wavelength of MI peak, the bandwidth of output spectrum can be greatly improved [22]. For the trigger signal to assist the spectral broadening, we set the frequency shift of the trigger signal to be $67 \mathrm{THz}$ (better spectral broadening) in the anomalous dispersion regime of the fiber and then vary the peak power of different duration trigger signals to investigate the characteristics (spectral broadening, coherence and SNR) of the final output SC signal. As for the comparison of coherence and SNR, the best choice of the frequency shift of the trigger signal is different from that of the frequency location better suited for spectral broadening. In this case, the suitable frequency shift of trigger signal is located near the MI peak but not exactly on the MI peak. After numerous simulations we figured out that $60 \mathrm{THz}$ to be more appropriate for improving the performance of SC signal coherence and SNR. Hence for all simulations related to the investigation of better coherence and SNR of the SC generation, we simply set the frequency shift of trigger to be $60 \mathrm{THz}$.

Quality of the coherence and SNR of the generated SC signal are respectively related to the phase stability and 

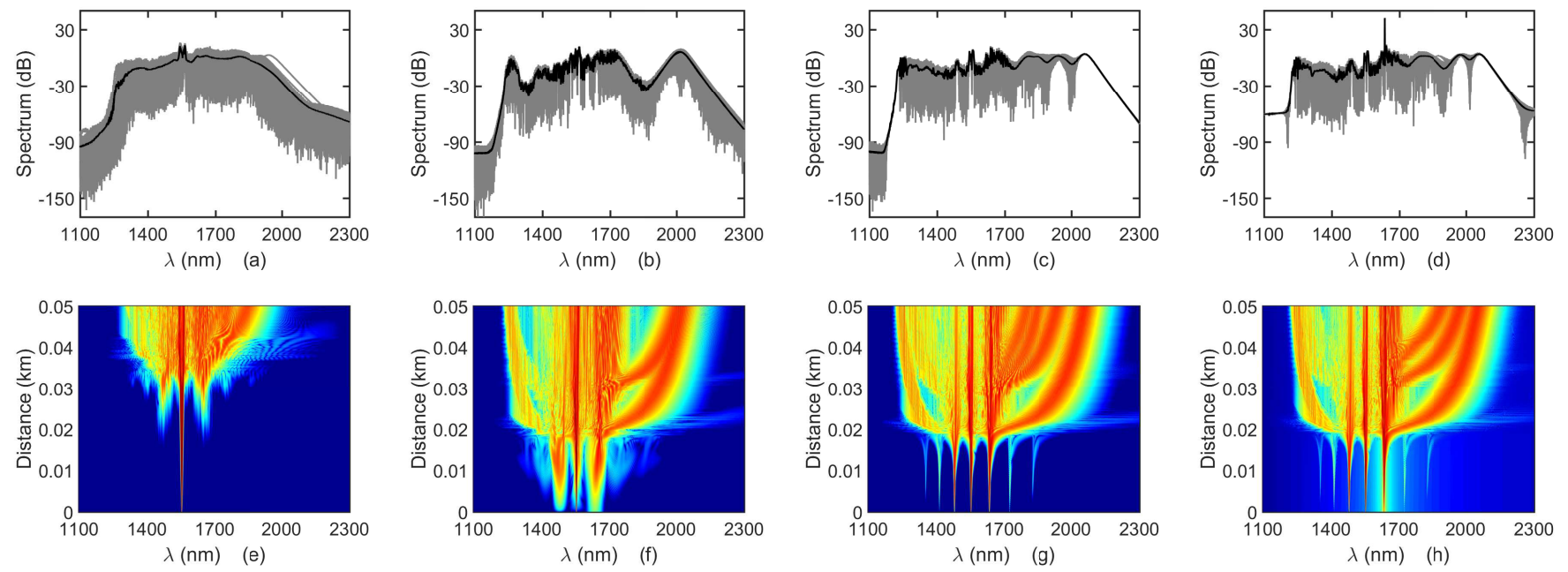

Fig. 3. Comparison between the SC signal spectra generated using (a) untriggered, (b) triggered by 100 fs pulse, (c) 4 ps pulse and (d) CW. For both triggered and untriggered SC generations, 100 individual simulations with different noise seeds are implemented where the gray lines stand for 100 simulations spectral output and black line stands for the calculated mean. (e), (f), (g), (h) show the one-shot simulation of spectral evolution for untriggered, 100 fs-triggered, 4 ps-triggered and CW-triggered spectrum respectively. The peak power of all three types of trigger signal is $1 \%$ that of the pump pulse.

intensity stability. Figure $1(\mathrm{c})$ and (d) depict the coherence and SNR of the untriggered SC. As shown in figure 1(c), the temporal coherence at the center wavelength $1554.5 \mathrm{~nm}$ is close to value 1 while at other wavelengths the values are poor. In order to quantify the overall coherence across the whole spectrum of SC, it is common to introduce a spectrally averaged overall coherence [2]. In case of the untriggered SC, the overall coherence for the wavelength range from $1300 \mathrm{~nm}$ to $2100 \mathrm{~nm}$ is calculated as 0.2987 . In figure $1(\mathrm{~d})$, we can find the SNR peal value as 4.888 at the pump center wavelength and for other wavelengths across the SC spectrum exhibit extremely low SNR values. Similar to the overall coherence, we can also define the overall SNR as reported in [7] to quantify overall intensity stability of the output SC spectrum. The overall SNR is calculated as 1.592 for the untriggered SC for the wavelength range from $1300 \mathrm{~nm}$ to $2100 \mathrm{~nm}$.

\section{B. Comparison of spectral broadening}

In order to understand the impact of the variation of the peak power of the trigger signal on the spectral broadening of ps pulse pumped SC generation, we set the frequency shift of the trigger signal to $67 \mathrm{THz}$ (best for spectral broadening) and then vary the peak power of the trigger signal from $0.0001 \%$ to $1 \%$ of the peak power of pump pulse. Figure 2 shows that fs pulse trigger (circle marks), ps pulse trigger (cross marks), or $\mathrm{CW}$ trigger (plus marks), all have the potential to greatly enhance the spectral broadening of ps pulse pumped SC generation. And as the peak power of the trigger increases, the effect of improving the SC bandwidth becomes more apparent.

From the results reported in figure 2, we select the appropriate peak power for the trigger signal to achieve the best results for spectral broadening in the further study of the output spectrum and spectral evolution. Figures 3(a), (b), (c) and (d) show the spectrum of untriggered, 100 fs-triggered, 4 pstriggered and $\mathrm{CW}$-triggered, ps pulse pumped $\mathrm{SC}$ generation. The peak power of all three types of trigger is selected to be $1 \%$ of that of the pump. As we have mentioned above, the spectrum of untriggered SC spans from $1276 \mathrm{~nm}$ to 2045 $\mathrm{nm}$ that corresponds to a bandwidth of $769 \mathrm{~nm}$. As shown in figure 3(b), (c) and (d), in contrast to the untriggered SC generation, all three types of trigger show bandwidth enhancement. The spectral range of 100 fs-triggered SC signal is from $1221 \mathrm{~nm}$ to $2208 \mathrm{~nm}$ (bandwidth of $987 \mathrm{~nm}$ ). The spectral range of 4 ps-triggered SC signal is from $1207 \mathrm{~nm}$ to $2236 \mathrm{~nm}$ (bandwidth of $1029 \mathrm{~nm}$ ). The spectral range of CWtriggered SC signal is from $1201 \mathrm{~nm}$ to $2243 \mathrm{~nm}$ (bandwidth of $1042 \mathrm{~nm}$ ). Out of the three types of trigger, CW trigger can achieve the best performance for the spectral broadening under the circumstance that the peak power of three types of trigger is the same. Figures 3(e), (f), (g) and (h) show the spectra of untriggered, 100 fs-triggered, 4 ps-triggered and $\mathrm{CW}$-triggered SC generation, respectively. The spectral broadening originates from accumulative $\mathrm{MI}$ that results in the formation of ps pulse into fundamental solitons. In the process of spectral broadening, the chromatic dispersion and some nonlinear effects such as Raman scattering, four-wave mixing, self-phase modulation and Kerr dispersion work together progressively to generate new spectral components. Therefore, the spectral broadening takes place continuously and finally gives rise to SC generation. As shown in figure 3(e), we can find that the untriggered SC has two side lobes on either side at around $25 \mathrm{~m}$. For the triggered cases, the first order MI continuously generates the second order which is assisted by four-wave mixing and goes on until it started broadening on the red side of the spectral evolution due to Raman scattering (Stokes). In the case of triggered SC generation, rather than the random noise seeds, the trigger signal actively participates with the MI signal in the process of four-wave mixing and therefore enhances the spectral broadening. Thus, the output spectrum of SC is wider and smoother when the pump pulse is added with a weak trigger signal. After the formation of ps pulse into fundamental solitons, the collisions of soliton 

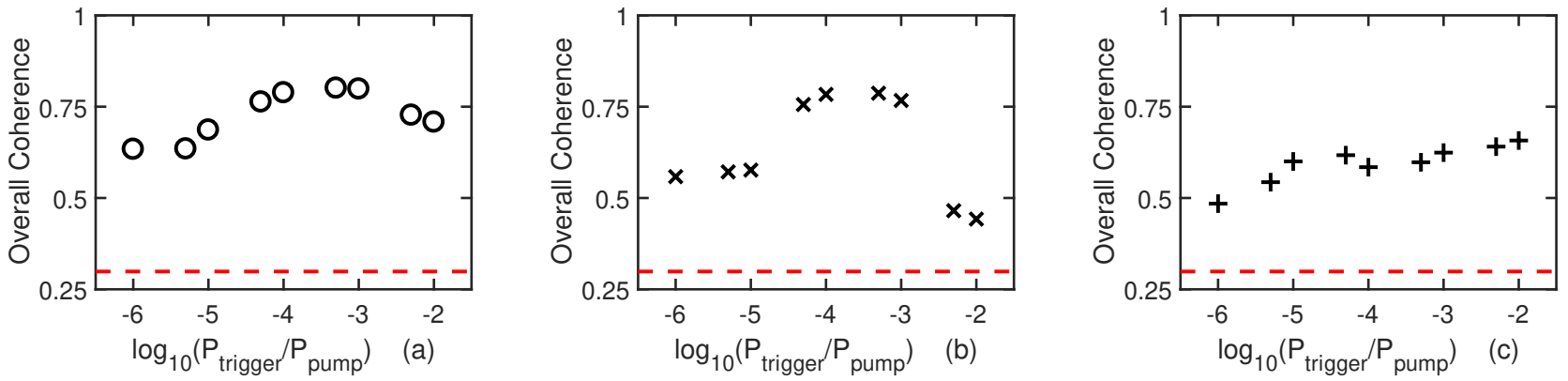

Fig. 4. Comparison of overall coherence between (a) 100 fs-triggered (circle marks), (b) 4 ps-triggered (cross marks), (c) CW-triggered (plus marks) and untriggered (red dashed line) SC generation. The peak power of triggers ranges from $0.0001 \%$ to $1 \%$ that of the pump pulse.
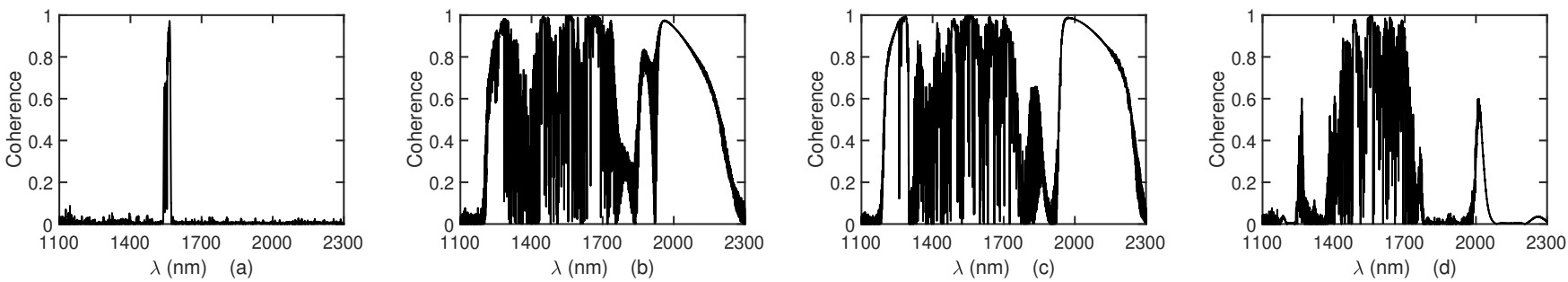

Fig. 5. Comparison of coherence between SC signal generated from (a) untriggered, (b) 100 fs-triggered, (c) 4 ps-triggered and (d) CW-triggered SC. The peak power of $100 \mathrm{fs}$ trigger, 4 ps trigger and $\mathrm{CW}$ trigger is $0.05 \%, 0.05 \%$ and $1 \%$ respectively.

lead to the formation of Raman soliton at longer wavelength regime. Meanwhile, dispersive waves are created at the shorter wavelength regime due to parts of solitons are blue-shifted. And we can find that the ps pulse trigger and $\mathrm{CW}$ trigger can more quickly cause the disintegration of ps pulse pump into fundamental solitons than in the case of fs pulse trigger according to figure 3(f), (g) and (h). Here, the trigger not only accelerates the process of MI by converting the ps pulse into fundamental solitons, but also reinforces the formation and stability of Raman solitons and dispersive waves which can considerably enhances the coherence and SNR of SC.

\section{Comparison of coherence}

In the previous subsection, we have demonstrated that a weak trigger can assist the formation of solitons, which can suppress the fluctuations of phase and intensity through coherent processes. Consequently, the phase and intensity stabilities can be enhanced in the SC generation. Therefore, adding a weak trigger not only improves the spectral broadening but also enhances the coherence and SNR. So we now investigate which type(s) and what level of peak power of triggers can lead to the best performance in improving the coherence of ps pulse pumped SC. For this study, we set the frequency shift of all three types of trigger as $60 \mathrm{THz}$ as this can result best performance for the coherence of the final output SC signal. Figure 4 shows the variation of overall coherence verses different values of the peak power of different types of trigger. The red dashed line stands for the overall coherence of untriggered SC signal, which equals to 0.2987. From figure 4 we can report that either fs pulse trigger or ps pulse trigger provides much better enhancement of the coherence of the SC signal than the $\mathrm{CW}$ trigger. In the process of $\mathrm{SC}$ generation, fs pulse trigger and ps pulse trigger have multiple wavelength components in the frequency domain that can stimulate and better assist the four-wave mixing process when the pump pulse (also having several spectral components) propagates along the optical fiber. While the $\mathrm{CW}$ has only one spectral component in the frequency domain so that the auxiliary effect of $\mathrm{CW}$ trigger on the four-wave mixing process is not as dominant as in the case of pulse type triggers. Therefore, the effect of CW trigger on coherence is not as good as fs pulse trigger or ps pulse trigger.

As shown in figure 4, the $\mathrm{CW}$ trigger derives the best performance for the coherence when the peak power of the trigger signal increased to $1 \%$ that of the pump pulse. While the fs pulse trigger or ps pulse trigger perform better on coherence than $\mathrm{CW}$ trigger even for much lower peak power values, which ranges from $0.005 \%$ to $0.1 \%$. Both fs pulse trigger and ps pulse trigger achieve their best performance on coherence at $0.05 \%$ peak power of trigger pulse with respect to pump signal.

Figure 5 shows the comparison of coherence between (a) untriggered, (b) 100 fs-triggered, (c) 4 ps-triggered and (d) $\mathrm{CW}$-triggered SC signal when the peak power of triggers are all fixed at their respective best performances. The peak power of $100 \mathrm{fs}$ trigger, $4 \mathrm{ps}$ trigger and $\mathrm{CW}$ trigger is $0.05 \%, 0.05 \%$ and $1 \%$ respectively. As for the untriggered SC generation case, the coherence at the center wavelength $1554.5 \mathrm{~nm}$ is close to 1 while that of other wavelengths are poor. The coherence of fs pulse triggered SC and ps pulse triggered SC signals is quite similar. For the 100 fs pulse triggered SC generation case, the coherence is enhanced, especially at the wavelengths range from around $1220 \mathrm{~nm}$ to $1740 \mathrm{~nm}$ and from around $1860 \mathrm{~nm}$ to $2130 \mathrm{~nm}$. The overall coherence at the 

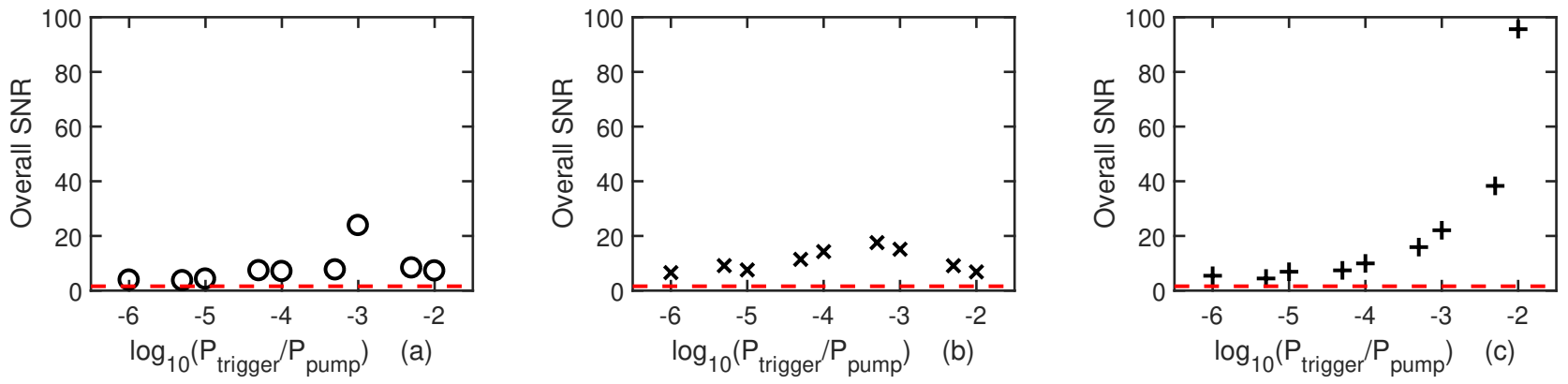

Fig. 6. Comparison of overall SNR between (a) 100 fs-triggered (circle marks), (b) 4 ps-triggered (cross marks), (c) CW-triggered (plus marks) and untriggered (red dashed line) in ps pulse pumped SC generation. The peak power of triggers ranges from $0.0001 \%$ to $1 \%$ that of the peak power of pump
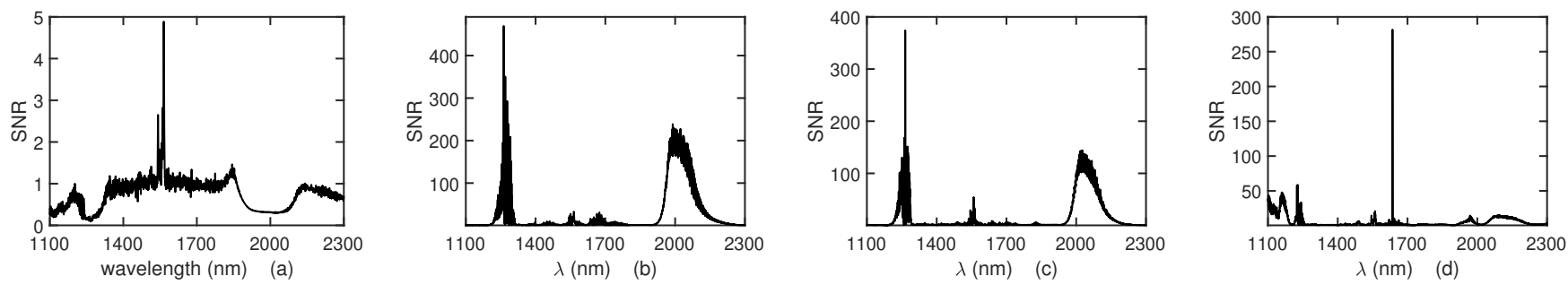

Fig. 7. Comparison of SC signal SNR between (a) untriggered, (b) 100 fs-triggered, (c) 4 ps-triggered and (d) CW-triggered SC. The peak power of 100 fs trigger, 4 ps trigger and $\mathrm{CW}$ trigger is $0.1 \%, 0.05 \%$ and $1 \%$ respectively, corresponding to the best performance of the SC signal spectral intensity SNR

wavelengths range from $1300 \mathrm{~nm}$ to $2100 \mathrm{~nm}$ is 0.8011 . And for the 4 ps pulse triggered SC generation case, the coherence is also greatly enhanced, especially at the wavelengths range from around $1200 \mathrm{~nm}$ to $1300 \mathrm{~nm}$, from around $1330 \mathrm{~nm}$ to $1750 \mathrm{~nm}$ and from around $1940 \mathrm{~nm}$ to $2190 \mathrm{~nm}$. The overall coherence at the wavelengths range from $1300 \mathrm{~nm}$ to 2100 $\mathrm{nm}$ is 0.7869 . Compared to the fs pulse trigger and ps pulse trigger, the $\mathrm{CW}$ trigger can only enhance the coherence of $\mathrm{ps}$ pulse pumped SC signal at the wavelengths range from around $1430 \mathrm{~nm}$ to $1690 \mathrm{~nm}$ and the overall coherence is 0.6574 and it requires twenty times more peak power compared to pulse triggered SC generation.

\section{Comparison of SNR}

Now we report the control of the SC signal spectral intensity stability by investigating the SNR of the ps pulse pumped SC generation under the influence of different types of trigger. For this study as well we fix the frequency shift of all three kinds of trigger as $60 \mathrm{THz}$ and then vary the peak power of the triggers from $0.0001 \%$ to $1 \%$ that of the peak power of pump. Figure 6 shows the variation of overall SNR against the range of peak power of different types of triggers. The red dashed line represents the overall SNR of untriggered SC generation that corresponds to 1.592. As shown in figure 6, adding a weak trigger signal can enhance the spectral intensity stability of ps pulse pumped SC generation compared to the untriggered process. We also find that as the peak power of triggers increased, the overall SNR of the CW triggered SC signal is better than those generated with the assistance of fs pulse trigger or ps pulse trigger. In both pulse-triggered cases, the performance of the overall SNR increases first and then decreases with the increase of the triggers' peak power. On the other hand, the overall SNR of CW triggered SC generated signal keeps increasing as the peak power of the trigger is increased.

Figures 7(a), (b), (c) and (d) show the comparison of SC signal spectral intensity SNR for the untriggered, fs pulse triggered, ps pulse triggered and $\mathrm{CW}$ triggered cases. The peak power of fs pulse trigger, ps pulse trigger and $\mathrm{CW}$ trigger is $0.1 \%, 0.05 \%$ and $1 \%$ respectively, which can best reinforce the spectral intensity stability of ps pulse pumped SC generation. Compared to the low values of SNR across the SC spectrum for the untriggered case in figure 7(a), the fs pulse trigger and ps pulse trigger dramatically pull the SNR to much higher values in both short wavelength and long wavelength ranges of the SC spectrum. As mentioned above, the overall SNR is defined as given in [7] to quantify overall intensity stability of output SC signal spectrum from $1300 \mathrm{~nm}$ to $2100 \mathrm{~nm}$. The overall SNR of the fs pulse triggered and ps pulse triggered SC is 23.848 and 17.556, while the overall SNR of the untriggered SC is only 1.592. In figure 7(d), The CW trigger pull the SNR values to high around the wavelength of $1635 \mathrm{~nm}$ where the trigger is added. The overall SNR of the $\mathrm{CW}$ triggered SC signal is 95.616 which is extremely high compared to the fs pulse triggered or ps pulse triggered SC generation. To achieve this high overall SNR value the required peak power of the $\mathrm{CW}$ trigger is ten times and twenty times larger than that of the fs pulse and ps pulse trigger cases, respectively.

\section{CONCLUSION}

In this paper, we have numerically demonstrated that weak fs pulse, ps pulse or CW trigger can help to improve the spectral broadening of SC generation and the feasibility to control the fluctuations of phase and intensity in the ps pulse 
pumped SC generation. Since the frequency shift and peak power of the triggers are the two main factors that influence the spectral broadening and stability of the SC signal, we have performed numerous simulations to determine that the frequency shift at $67 \mathrm{THz}$ is best for the spectral broadening case and $60 \mathrm{THz}$ is best for the best performances for both coherence and SNR. Therefore, we fixed the frequency shift of the triggers and varied the peak power of the triggers to figure out the most effective choice. In the case of spectral broadening, all three types of trigger can greatly enhance the bandwidth of the SC spectrum as the peak power of the triggers is increased. In the case of coherence, fs pulse trigger and ps pulse trigger achieve better at a much lower peak power than $\mathrm{CW}$ trigger. However, both the fs pulse trigger and ps pulse trigger require precise control of the time delay between pump and trigger, which is still difficult in terms of the actual experiment. We believe the simulation results reported here can provide good guidance and pave the way to conduct experiments on SC generation by selecting the proper signal duration and suitable peak power for the trigger.

\section{REFERENCES}

[1] S. Martin-Lopez, M. Gonzalez-Herraez, P. Corredera, M. L. Hernanz, and A. Carrasco, "Gain-flattening of fiber raman amplifiers using nonlinear pump spectral broadening," Optics Communications, vol. 242, no. 4-6, pp. 463-469, 2004.

[2] J. M. Dudley, G. Genty, and S. Coen, "Supercontinuum generation in photonic crystal fiber," Reviews of Modern Physics, vol. 78, pp. 1135$1184,2006$.

[3] W. Brian and N. Nathan, "Phase, timing, and amplitude noise on supercontinua generated in microstructure fiber," Optics Express, vol. 12, no. 10 , pp. 2166-75, 2004.

[4] D. L. Marks, A. L. Oldenburg, J. J. Reynolds, and S. A. Boppart, "Study of an ultrahigh-numerical-aperture fiber continuum generation source for optical coherence tomography," Optics Letters, vol. 27, no. 22, pp. 2010-2012, 2002.

[5] D. R Solli, C. Ropers, and B. Jalali, "Active control of rogue waves for stimulated supercontinuum generation," Physical Review Letters, vol. 101 , p. 233902, 2009.

[6] G. Genty and J. M. Dudley, "Route to coherent supercontinuum generation in the long pulse regime," IEEE Journal of Quantum Electronics, vol. 45, no. 11, pp. 1331-1335, 2009.

[7] S. T. Sørensen, C. Larsen, U. Møller, P. M. Moselund, C. L. Thomsen, and O. Bang, "Influence of pump power and modulation instability gain spectrum on seeded supercontinuum and rogue wave generation," Journal of the Optical Society of America B, vol. 29, no. 10, pp. 2875$2885,2012$.

[8] C. Huang, H. Y. Fu, and Q. Li, "Stimulated supercontinuum generation in CW-pumped regime," in Frontiers in Optics / Laser Science, JTu2A.42, 2018.

[9] M. Maus, E. Rousseau, M. Cotlet, G. Schweitzer, J. Hofkens, M. V. D. Auweraer, F. C. D. Schryver, and A. Krueger, "New picosecond laser system for easy tunability over the whole ultraviolet/visible/near infrared wavelength range based on flexible harmonic generation and optical parametric oscillation," Review of Scientific Instruments, vol. 72, no. 1, pp. 36-40, 2001.

[10] C. Lin and R. H. Stolen, "New nanosecond continuum for excited-state spectroscopy," Applied Physics Letters, vol. 28, no. 4, pp. 216-218, 1976.

[11] A. Rulkov, M. Vyatkin, S. Popov, J. Taylor, and V. Gapontsev, "High brightness picosecond all-fiber generation in $525-1800 \mathrm{~nm}$ range with picosecond Yb pumping," Optics Express, vol. 13, no. 2, pp. 377-81, 2005.

[12] M. Okuno, H. Kano, P. Leproux, V. Couderc, and H.-O. Hamaguchi, "Ultrabroadband $\left(>2000 \mathrm{~cm}^{-1}\right)$ multiplex coherent anti-Stokes Raman scattering spectroscopy using a subnanosecond supercontinuum light source," Optics Letters, vol. 32, pp. 3050-2, 2007.
[13] J. M. Dudley, G. Genty, F. Dias, B. Kibler, and N. Akhmediev, "Modulation instability, Akhmediev Breathers and continuous wave supercontinuum generation," Optics Express, vol. 17, pp. $21497-508$, 2009.

[14] K. Tai, A. Tomita, J. L. Jewell, and A. Hasegawa, "Generation of subpicosecond solitonlike optical pulses at $0.3 \mathrm{THz}$ repetition rate by induced modulational instability," Applied Physics Letters, vol. 49, no. 5, pp. 236-238, 1986.

[15] A. S. Gouveia-Neto, M. E. Faldon, and J. R. Taylor, "Raman amplification of modulational instability and solitary-wave formation," Optics Letters, vol. 13, no. 11, pp. 1029-31, 1988.

[16] D. R. Solli, C. Ropers, P. Koonath, and B. Jalali, "Optical rogue waves," Nature, vol. 450, no. 7172, pp. 1054-1057, 2007.

[17] A. Mussot, A. Kudlinski, M. I. Kolobov, E. Louvergneaux, and M. Taki, "Convective instabilities and optical rogue waves in fibers with $\mathrm{CW}$ pumping," in IEEE/LEOS Winter Topicals Meeting Series, TUC2.5, 2009.

[18] C. Lafargue, J. Bolger, G. Genty, F. Dias, J. M. Dudley, and B. J. Eggleton, "Direct detection of optical rogue wave energy statistics in supercontinuum generation," Electronics Letters, vol. 45, no. 4, pp. 217 219, 2009.

[19] M. Taki, A. Mussot, A. Kudlinski, E. Louvergneaux, M. Kolobov, and M. Douay, "Third-order dispersion for generating optical rogue solitons," Physics Letters A, vol. 374, no. 4, pp. 691-695, 2010.

[20] A. Aalto, G. Genty, and J. Toivonen, "Extreme-value statistics in supercontinuum generation by cascaded stimulated Raman scattering," Optics Express, vol. 18, no. 2, pp. 1234-1239, 2010.

[21] K. K. Y. Cheung, Z. Chi, Z. Yue, K. K. Y. Wong, and K. K. Tsia, "Manipulating supercontinuum generation by minute continuous wave," Optics Letters, vol. 36, no. 2, pp. 160-2, 2011.

[22] Q. Li, F. Li, K. K. Y. Wong, A. P. T. Lau, K. K. Tsia, and P. K. A. Wai, "Investigating the influence of a weak continuous-wave-trigger on picosecond supercontinuum generation," Optics Express, vol. 19, no. 15, p. 13757, 2011.

[23] O. Vanvincq, B. Barviau, A. Mussot, G. Bouwmans, Y. Quiquempois, and A. Kudlinski, "Significant reduction of power fluctuations at the long-wavelength edge of a supercontinuum generated in solid-core photonic bandgap fibers," Optics Express, vol. 18, no. 23, pp. $24352-$ 24360,2010

[24] J. M. Dudley, G. Genty, and B. J. Eggleton, "Harnessing and control of optical rogue waves in supercontinuum generation," Optics Express, vol. 16, no. 6, pp. 3644-3651, 2008.

[25] G. Genty, J. M. Dudley, and B. J. Eggleton, "Modulation control and spectral shaping of optical fiber supercontinuum generation in the picosecond regime," Applied Physics B, vol. 94, no. 2, pp. 187-194, 2009.

[26] J. M. Dudley, F. Dias, M. Erkintalo, and G. Genty, "Instabilities, breathers and rogue waves in optics," Nature Photonics, vol. 8, no. 10, pp. 755-764, 2014.

[27] D. M. Nguyen, T. Godin, S. Toenger, Y. Combes, B. Wetzel, T. Sylvestre, J.-M. Merolla, L. Larger, G. Genty, F. Dias, and J. M. Dudley, "Incoherent resonant seeding of modulation instability in optical fiber," Optics Letters, vol. 38, no. 24, pp. 5338-5341, 2013.

[28] R. Zhou, R. Huang, Q. Li, and H. Y. Fu, "Raman soliton at $2 \mu \mathrm{m}$ in picosecond pumped supercontinuum by a weak CW trigger," Optics Express, vol. 27, no. 9, pp. 12976-12 986, 2019.

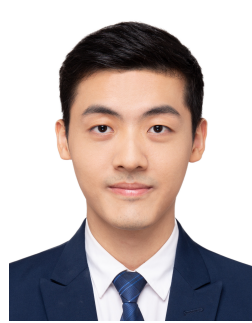

Chao Huang received the Bachelor of Science degree from Sun Yat-sen University, Guangzhou, China, in 2017. He was the vice president of OSA Student Chapter in Peking University Shenzhen Graduate School in 2017-2018. He is currently pursuing the M.S. degree with the School of Electronic and Computer Engineering, Peking University. 


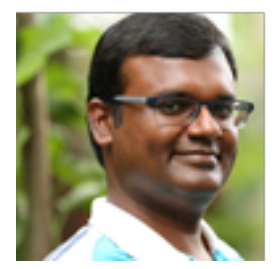

K. Nakkeeran (Ph.D., CEng, SMIEEE, SMOSA, MIET) received the B.Eng. degree from the Coimbatore Institute of Technology, Coimbatore, India, in 1993, and the M.Tech. and Ph.D. degrees from Anna University, Chennai, India, in 1995 and 1998, respectively. In 1999, he joined the Institute of Mathematical Sciences, Chennai, where he was a Post-Doctoral Fellow for ten months. In 1999, he became a Research Associate with the Department of Physics, University of Burgundy, Dijon, France. In 2002, he became a Post-Doctoral Fellow with the Department of Electronic and Information Engineering, The Hong Kong Polytechnic University. In 2005, he joined the School of Engineering, University of Aberdeen, Aberdeen, U.K., where he has been a Senior Lecturer since 2011. His research interests include solitons, fiber lasers, fiber sensors, modeling and simulations of optical devices, long-haul optical fiber communications, and nonlinear science.

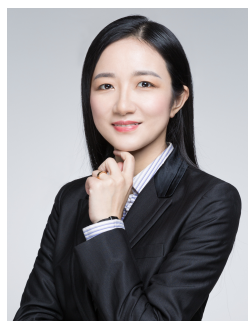

Qian Li (Ph.D., MIEEE, SMOSA) received the Bachelor of Science degree from Zhejiang University, Hangzhou, China, in 2003, the Master of Science degree from the Royal Institute of Technology (KTH), Stockholm, Sweden, in 2005, and the Ph.D. degree from the Hong Kong Polytechnic University, Hong Kong, in 2009. After graduation she was a Visiting Scholar at the University of Washington, Seattle and Postdoctoral Fellow at the Hong Kong Polytechnic University. In 2012 she joined School of Electronic and Computer Engineering (ECE) in Peking University as an Assistant professor. Since 2013 she is Associate Professor at ECE. Her research interests include nonlinear optics, ultrafast optics and integrated optics. Dr. Li is members of Institute of Electrical and Electronics Engineers (IEEE) and a senior member of the Optical Society of America (OSA). From March 2017 to April 2019, she was Vice Chair of IEEE ED/SSC Beijing Section (Shenzhen) Chapter and Chair for EDS. From 2015 she is an advisor of OSA Student Chapter in Peking University Shenzhen Graduate School. From 2019, she is an advisor of Peking University Shenzhen Graduate School Photonic Society Student Branch Chapter. 